\title{
Use of single-mode optical fiber in the stabilization of laser frequency
}

\author{
Ying T. Chen
}

\begin{abstract}
A new method of using a Mach-Zehnder interferometer formed by single-mode optical fibers to stabilize the frequency of a helium-neon laser has been studied. Preliminary experimental result of $5000-\mathrm{Hz}$ linewidth within the time scale of $1 \mathrm{~s}$ is presented.
\end{abstract}

\section{Introduction}

For the purpose of obtaining very high precision short term frequency stabilization of laser beams, an optical resonator has proved to be a powerful tool to provide frequency discrimination to control the gas laser. Since Drever et $a l^{1}$ proposed and developed a new technique using an improved rf sideband type of optical discriminator to stabilize the laser phase and frequency, the cavity stabilizer has been applied widely in various fields.

A new fiber optic stabilizer capable of locking the frequency of low power laser beams within a medium time scale is studied in this paper. This stabilizer is much more simple and flexible than the others.

First, the use of a fiber interferometer with unequal arms as a frequency phase converter is discussed. Then an experimental setup to stabilize a helium-neon laser is described. As the present experiment has not yet reached the limit of precision, further improvement is expected although the mechanical thermal noise may give serious limitation for the application of fiber optics in stabilizing the frequency of a laser to higher precision, i.e., linewidth of a few hertz.

\section{Working Mechanism}

The operating schematic is shown in Figs. 1 and 2. After an optical isolator, the laser beam is first divided by a beam splitter, the transmitted part from the beam splitter is used to stabilize the laser. This beam then couples to a single-mode fiber directional coupler which provides beam splitting. The two tails of this

The author is with California Institute of Technology, Division of Physics, Mathematics \& Astronomy, Pasadena, California 91125. Received 15 June 1988.

0003-6935/89/112017-05\$02.00/0.

(C) 1989 Optical Society of America. directional coupler are spliced to two single-mode fibers with optical lengths of $L_{1}$ and $L_{2}$, respectively. A PZT driven by the high frequency reference signal is wrapped by fiber 2 to produce the phase modulation. We assume that the electric fields of the radiation before combining at the second beam splitter are $E_{1}$ and $E_{2}$, respectively. Thus, if $L_{1} \gg L_{2}$, we have

$$
\begin{aligned}
& E_{1}=E_{0} \sin \left(\Omega^{\prime} t+\Omega^{\prime} t_{0}\right), \\
& E_{2}=E_{0} \sin (\Omega t+\Gamma \sin \omega t),
\end{aligned}
$$

where $\Omega^{\prime}, \Omega$ are the optical frequencies at the ends of fibers 1 and $2, \Gamma$ and $\omega$ are the amplitude and frequency modulations, and

$$
t_{0}=\left(L_{1}-L_{2}\right) / c \text {, }
$$

where $c$ is the speed of light. After these two beams are combined, provided the polarization of the beams in both fibers can be kept, the electric field is

$$
E=E_{1}+E_{2} \text {. }
$$

If we rewrite $E_{2}$ in Eq. (4) using the Bessel series approximation, the radiation intensity incident on the photodetector can be calculated as follows:

$$
\begin{aligned}
I= & |E|^{2} \\
= & E_{0}^{2}\left\{2+\Gamma / 2-\Gamma / 2 \cos 2 \omega t+2 \cos \left[\left(\Omega-\Omega^{\prime}\right) t-\Omega^{\prime} t_{0}\right]\right. \\
& +\Gamma \cos \left[\left(\Omega-\Omega^{\prime}\right) t+\omega t-\Omega^{\prime} t_{0}\right] \\
& \left.-\Gamma \cos \left[\left(\Omega-\Omega^{\prime}\right) t-\omega t-\Omega^{\prime} t_{0}\right]\right\} .
\end{aligned}
$$

The above signal will be demodulated in the lock-in amplifier and it is easy to prove that after filtering the high frequency components of the output from the mixer, the output of the lock-in amplifier becomes,

$$
P=-E_{0}^{2} \Gamma \sin \left[\left(\Omega-\Omega^{\prime}\right) t-2 \pi \frac{L_{1}-L_{2}}{\lambda^{\prime}}\right],
$$

where $\lambda^{\prime}$ is the optical wavelength of the light at the end of fiber 1 . This is the error signal which will be 


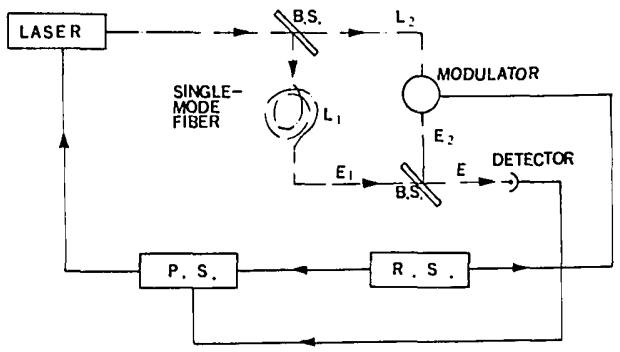

Fig. 1. Scheme of the optical fiber used as optical delay line to stabilize the frequency of laser. In the drawing, R.S. means reference signal; P.S. means phase sensitive detector; B.S. means beam splitter.

used to feed back to the laser cavity thus stabilizing the frequency. After signal $P$ is fed back, the phase of the laser is locked, i.e., with the feedback loop closed, and we have

$$
\sin \left[\left(\Omega-\Omega^{\prime}\right) t-2 \pi \frac{L_{1}-L_{2}}{\lambda^{\prime}}\right]=0 .
$$

The solution that satisfies Eq. (7) is

$$
\left(\Omega-\Omega^{\prime}\right) t-2 \pi \frac{L_{1}-L_{2}}{\lambda^{\prime}}=n \pi,
$$

where $n$ is an integer. If $\left(L_{1}-L_{2}\right)$ is assumed to be constant, Eq. (8) may be further separated in the following way:

$$
\begin{aligned}
\Omega-\Omega^{\prime} & =0, \\
2 \frac{L_{1}-L_{2}}{\lambda^{\prime}} & =n \pi .
\end{aligned}
$$

This is so because $t$ in Eq. (8) is a variable. Equation (9.2) shows that $\left(L_{1}-L_{2}\right)$ can be used to define $\lambda^{\prime}$ and Eq. (9.1) shows that with such a feedback the frequency of light at different points of the optical fiber will follow the defined frequency for any time of $t$. This explains the principle of stabilizing the frequency of the laser by using an optical fiber.

\section{Noise Study}

It is obvious that in practice $\left(L_{1}-L_{2}\right)$ is not constant. Therefore different kinds of noise may occur. Generally speaking, the noise can be investigated in such a way that in Eq. (8) the length $\left(L_{1}-L_{2}\right)$ is a function of time and, moreover without losing generality, we have

$$
L_{1}-L_{2}=L_{1 o}-L_{2 o}+\left(L_{1 o}-L_{20}\right) \sum B_{i} \sin \left(m_{i} t\right),
$$

where $B_{i}$ is the amplitude of the relative change of the length difference, $m$ is the circular frequency of the fluctuation, $L_{1 o}$ and $L_{2 o}$ are the average values of $L_{1}$ and $L_{2}$. Therefore, for the fluctuation of a certain frequency $m$, instead of Eq. (9), we will have

$$
\begin{aligned}
\left(\Omega-\Omega^{\prime}\right) t \lambda^{\prime} & =2 \pi\left(L_{1 o}-L_{2 o}\right) B \sin (m t), \\
2 \frac{L_{1}-L_{2}}{\lambda^{\prime}} & =n \pi .
\end{aligned}
$$

It is easy to derive from Eq. (11.1) that, in time scale $t>$

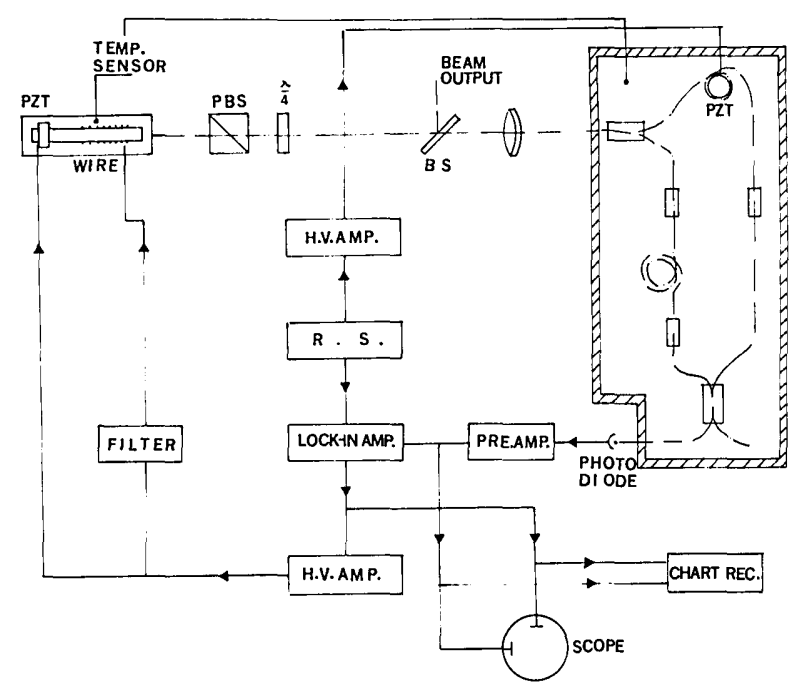

Fig. 2. The experimental setup of the fiber optics stabilizer. All the parts of the optical frequency-phase convertor are housed in a temperature controlled cylinder which is sealed in a vacuum tank. In the drawing, PBS means polarized beam splitter; H.V. AMP means high voltage amplifier.

$t_{0}=\left(L_{1 o}-L_{2 o}\right) / c$, the maximum possible frequency change is

$$
\delta f / f=B \equiv \frac{\delta\left(L_{1}-L_{2}\right)}{L_{1}-L_{2}} .
$$

Therefore, we conclude the simple result that the fluctuation of the optical length of fiber is linearly proportional to the fluctuation of frequency. This result will help us to study the various sources of noise.

For a low output level laser, we may omit the noise caused by nonlinear scattering processes, so the noise source could be temperature drift, shot noise, mechanical thermal noise, vibration, acoustic noise, strain noise, etc. In the following, we will only concentrate on discussing the first three because the temperature drift could be a very large noise, the shot noise and thermal noise have their intrinsic characters which will give some theoretical limitation to the ultimate precision. The other noise listed above could be reduced by the practical isolation as described in Sec. IV.A.

\section{A. Temperature Drift of Frequency}

If we assume that the optical fiber has been settled without stress including the stress caused by external acoustic noise, the main source of external noise will be the temperature fluctuation. Due to the environmental temperature change $d T$, the increment of optical length of a single-mode fiber with length $l$ and core refractive index $n$ can be expressed as

$$
d L=n \frac{\partial l}{\partial T} d T+l \frac{\partial n}{\partial T} d T .
$$

Therefore the frequency drift of the laser beam in the scheme described in Sec. II during the time period when the temperature changes $d T$, according to Eq. (12), is 


$$
\delta f / f=B=\frac{1}{n_{1} l_{1}-n_{2} l_{2}}\left(l_{1} \frac{\partial n_{1}}{\partial T}-l_{2} \frac{\partial n_{2}}{\partial T}+n_{1} \frac{\partial l_{1}}{\partial T}-n_{2} \frac{\partial l_{2}}{\partial T}\right) d T,
$$

where $l_{1}$ and $l_{2}$ are the lengths of fiber 1 and fiber 2 respectively. It is obvious from the above equation that there are two ways to diminish the instability of the frequency of light caused by the temperature change. First, we can keep the temperature fluctuation $d T$ as small as possible. Second, different fiber materials can be used so that the value in parentheses remains as small as possible. However, an interesting fact worthy of note is that when the same material is used for both arms, as we know, for ordinary singlemode fiber, we have

$$
\frac{1}{l} \frac{\partial l}{\partial T} \cong 5 \times 10^{-7} \mathrm{~K}^{-1}, \quad \frac{\partial n}{\partial T} \cong 10^{-5} \mathrm{~K}^{-1} .
$$

Equation (14) can be rewritten approximately as

$$
\delta f / f \cong \frac{1}{n} \frac{\partial n}{\partial T} d T .
$$

This result shows that, when the same materials are used for both fibers $l_{1}$ and $l_{2}$, generally speaking the temperature effect is independent of the length $\left(l_{1}-\right.$ $\left.l_{2}\right)$. On the other hand, increasing the length of $\left(l_{1}-\right.$ $l_{2}$ ) gives a larger error signal, because the phase change of the electric field at the point of combination in Fig. 1 is proportional to the difference of optical length $\left(L_{1}-\right.$ $L_{2}$ ). This means that in practice a relatively long fiber can be used without increasing the effect of the temperature change. As an example, if we assume the daily temperature change for the fiber is $0.02 \mathrm{~K}$, the daily frequency fluctuation of the laser is $\sim 10^{-7}$, the fluctuation within $1 \mathrm{~s}$ is $3 \times 10^{-12}$. Actually, it is not difficult to control the temperature of the environment of fiber to make $d T$ much smaller than the above example figure.

\section{B. Photoelectron Shot Noise}

Shot noise in the photocurrent is one of the wellknown fundamental limitations for the sensitivity of interferometers. The power spectral density of the fluctuation of the laser frequency due to shot noise is

$$
(\delta f / f)_{\omega}=\frac{1}{L_{1}-L_{2}} \sqrt{\frac{\hbar c \lambda^{\prime}}{\pi \eta p}},
$$

where $\hbar$ is Planck's constant, $c$ is speed of light, $\eta$ is the quantum efficiency of the photodiode, and $p$ is the light power. For typical values $(\eta p=0.1 \mathrm{mw}, \lambda=6.3 \times$ $10^{-5} \mathrm{~cm}, L_{1}-L_{2}=25 \mathrm{~m}$ ) we obtain a limited theoretical frequency instability of $(\delta f / f)_{\omega}=2 \times 10^{-16} / \sqrt{\mathrm{Hz}}$. Compared with other noise sources, shot noise is not the major one in the experiment described here.

\section{Intrinsic Thermal Noise}

Thermal noise is another theoretical limitation for the frequency stabilization. This noise occurs due to the fluctuations of both the length and the density of the fiber; the change of the density of fiber further changes the refractive index. Statistically these two kinds of fluctuation are independent, so the following discussion only concentrates on the thermal noise caused by the statistical fluctuation of the density. On the other hand the length fluctuation can be discussed in a similar manner.

The following result is calculated under the assumption that the system considered is uniform. The optical fiber is typically made of silica with dopants added to the core to increase the index of refraction over that of the cladding by $\sim 1 \%$. Because the nonuniformity is not great it is possible to model the optical fiber as a uniform solid wire to obtain an estimate of the order of the mechanical thermal noise. Because this paper is concerned mainly with a stabilizer of medium time scale, the following calculation is only for the low frequency range, i.e., $1-100 \mathrm{~Hz}$.

The mean value of the fluctuation of the density $\rho$ of a single-mode fiber of length $l$ and cross section $A$ can be expressed as

$$
\left(\overline{\delta \rho}^{2}\right)=\rho^{2} \gamma \frac{k T}{l A},
$$

where $k$ is Boltzmann's constant and

$$
\gamma=-\frac{1}{V}\left(\frac{\partial V}{\partial p}\right)_{T}
$$

is the compression coefficient of the fiber material. From the above equation, according to Landau and Lifshitz ${ }^{2}$ the power spectral density of the fluctuation density takes the following form:

$$
(\delta \rho)_{\omega}^{2}=2 \rho^{2} \beta \gamma \frac{k T}{l A} \frac{1}{\omega^{2}+\beta^{2}},
$$

where $\omega$ is the frequency, $\beta$ is a damping factor which may be approximately estimated as $\beta=v / l$, with $v$ as the speed of sound in the fiber and $l$ as the length of the fiber.

We use the linear measurement $(\delta \rho)_{\omega}=\sqrt{(\delta \rho)_{\omega}^{2}}$ to calculate the fluctuation of density. Due to the linear relationship between the density and refractive index, the strain noised caused by the fluctuation of fiber density can be calculated. The frequency noise in the experiment described by Fig. 1 has the order of

$$
(\delta f / f)_{\omega}=\frac{\sqrt{2}}{l} \sqrt{\left.\frac{k T \gamma v}{A\left(\omega^{2}+v^{2} / l^{2}\right.}\right)} .
$$

From the above equation we conclude that, for low frequency $\omega \ll v / l$, the thermal noise is white noise which represents the worst case; also in this case, the noise is independent of the length. For typical values $\left(v=5 \times 10^{5} \mathrm{~cm} / \mathrm{s} A=1.2 \times 10^{-4} \mathrm{~cm}^{2}, T=300 \mathrm{~K}, \gamma=2 \times\right.$ $10^{-12} \mathrm{~cm}^{2} / \mathrm{dgn}$ ), we obtain an order of limited theoretical frequency stability of

$$
(\delta f / f)_{\omega}=5 \times 10^{-14} / \sqrt{\mathrm{Hz}} .
$$

The limited noise estimated here is only one component of many possible noise sources- that due to thermal fluctuation in fiber density alone. Other thermal noise components arise from fluctuation in fiber length or the diameter. Following the same method as above, ideally length fluctuation will have the same order as the effect on the noise contribution. But in practice, it may be larger than the effect of density fluctuation because the length fluctuation may depend on how the 
fiber is supported and shrouded. Also the thermal noise of the frame housing the fiber could make considerable contribution to the noise. However, we do not attempt to consider such contributions here in detail, we just present the thermal induced density fluctuation as an example of one of several possible theoretical lower limits to achievable noise. In addition, we must emphasize that the noise sources studied above are not the only important sources; other external noise could be very important. For example, the microphonic effects can be important if the fiber is in air; mechanical motion of the fiber supporting structure may be significant, etc. But this kind of external noise can be limited by very careful isolation of the fiber as is discussed in Sec. IV.

Summarizing the above theoretical studies, we found that using optical fiber is probably a feasible method to stabilize the frequency of gas lasers with a linewidth of a few hundred hertz to a few hertz. In addition, it is worth mentioning that, without any moving part, optical fiber stabilization suffers much less mechanical vibration than other methods. Also, other advantages such as small volume, easy alignment, and low cost make this method particularly attractive. Fiber optics may be useful in stabilizing the frequency of laser beams when the medium time scale (seconds to a few hundreth second) stability is specially required.

\section{Experiment}

\section{A. Layout of the Experiment}

The experimental setup shown in Fig. 2 is used to control the frequency of a helium-neon laser. Divided by a beam splitter, part of the beam is used for stabilizing the laser. This beam, after passing an optical isolator, enters into a precision fiber coupler and a single-mode fiber directional coupler which provides 1:1 ratio low loss beam splitting. Two beams then pass through two different lengths of single-mode fibers (with a length difference of $25 \mathrm{~m}$ ). One of the fibers wraps around a $5-\mathrm{cm}(2-\mathrm{in}$.) diam PZT which is driven by a high voltage ac signal to produce phase modulation; the modulation frequency is $50 \mathrm{kHz}$. The beams recombine in a second fiber directional coupler to form a Mach-Zehnder type interferometer.

The signal detected by a fiber optic detector is first amplified in a lock-in amplifier (with a time constant of $3 \mathrm{~ms}$ ) and then by a high voltage amplifier. The signal is fed back to the laser in the same manner as that used by Baer et $a .^{3}$ and Zumberge. ${ }^{4}$ A hollow PZT which is attached firmly to the tube can be driven by the feedback error signal to stretch the length of the laser cavity, thus changing the frequency of the laser. Also, the same error signal is integrated (with a time constant of $2 \mathrm{~s}$ ) to control a heater which is wrapped around the tube to provide a larger and slower frequency correction.

Having good insulation, including vibrating, thermal, and acoustic insulation, is the key issue of this experiment. Because the fiber has a relatively small volume, this could be done in an easy and inexpensive

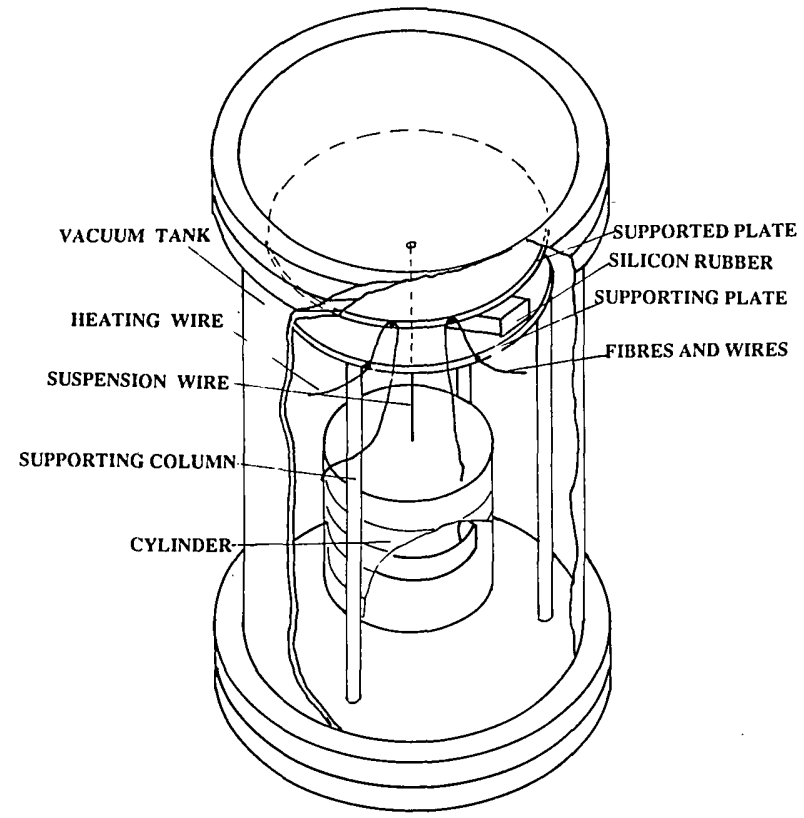

Fig. 3. Schematic illustration of the mechanical setup of optical fiber stabilizer.

way. In our experiment the fiber interferometer and associated parts are placed in a small box which is housed in a metal cylinder. Electrical heating wire is wrapped on the surface of the cylinder to provide an active temperature control. This cylinder is then hung by a suspension wire fixed to a supported plate as shown in Fig. 3. To achieve better isolation, this plate is supported through a layer of silicon rubber by a supporting plate. Figure 3 shows a detailed illustration of the insulation structure. All these packages are sealed in a vacuum tank. To avoid the vibration caused by the pumping system, the pumping port is closed and disconnected from the pipe. The vacuum chamber is made of stainless steel and the two surfaces are well polished to reduce temperature fluctuation by heat radiation. The active temperature control can stabilize the temperature of the cylinder to within $0.05^{\circ} \mathrm{C} /$ day.

\section{B. Experimental Result}

To measure the reproducibility of the stabilized laser, a heterodyne method can be used.5,6 This method requires two stabilized lasers which are not available at this laboratory. Instead, a new fiber interferometer housed in the same well-insulated environment is employed in the present experiment to measure the residual frequency fluctuation of the laser. This second fiber interferometer has a greater length difference than the first $(35 \mathrm{~m})$. First, we must verify that this method is able to detect the residual frequency fluctuation, because as we can easily guess, with two interferometers housed in the same environment, some kinds of fluctuation may cancel each other.

To investigate this problem, we assume that the first fiber interferometer used as a frequency phase converter has an optical length difference $L_{a}$, the second, 


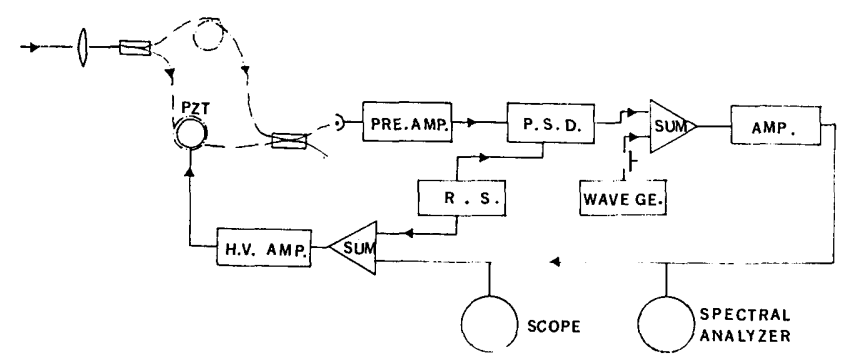

Fig. 4. The control diagram of the testing fiber interferometer.

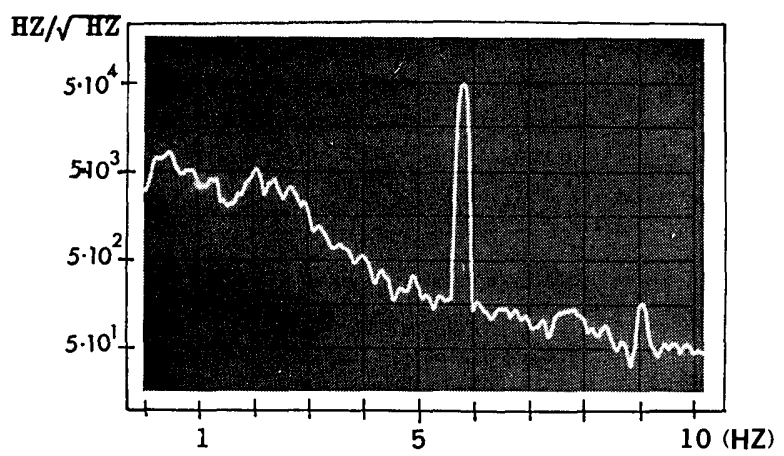

Fig. 5. Power spectrum of the fluctuation of laser output taken when the fiber stabilizer is working and the output of the testing fiber interferometer is locked on the dark fringe. The high peak in the photo is the calibration signal.

used as the testing interferometer, has an optical length difference $L_{b}$. Because of the external noise, they have optical length increments of $d L_{a}$ and $d L_{b}$, respectively. The frequency change of the laser due to the optical length change of the first interferometer is given by

$$
\delta f / f=d L_{a} / L_{a},
$$

and the second interferometer will have a phase change of

$$
\phi=2 \pi \frac{d L_{b}}{\lambda}\left(1-\frac{d L_{a} \times L_{b}}{d L_{b} \times L_{a}}\right) .
$$

Therefore, we conclude that the proportional change of the length differences will be canceled. Among all the sources of noise, only temperature drift can produce this type of proportional change. However as discussed before the frequency drift caused by the temperature change is well understood and easily calculated from the temperature measurement of the environment.
As shown in Fig. 4, the output of the phase sensitive detector is used to feed back the PZT to keep the fringe in the photodetector dark. This feedback voltage is then measured by a spectrum analyzer to obtain a spectrum of the fluctuation of the frequency of the laser as shown in Fig. 5. The calibration procedure is as follows. First, we open the first control loop, i.e., the laser beam is not stabilized, and open the second feed back as well. In this case, a maximum output of $15 \mathrm{~V}$ was observed in the scope of Fig. 4, which represents one fringe change in the photodetector of the testing fiber interferometer or, equivalently, a frequency change of $3 \mathrm{MHz}$ in the laser beam. Then we stabilize the laser beam, close the feedback loop of the testing interferometer, and turn on the wave generator to provide a signal of $6 \mathrm{~Hz}$ to the PZT as a calibration signal. The amplitude of the calibration was adjusted so that it gave a reading of $0.25 \mathrm{~V}$ in the same scope which represents a frequency change of $50 \mathrm{kHz}$ in the laser beam. The measured result shows that the frequency of the laser has an instability of $\left\langle\delta f / f=10^{-11}\right.$ within a time period of $1 \mathrm{~s}$.

The author is grateful to $R$. Drever for suggesting this project and his stimulating discussion and earnest criticism; to S. Vass for his technical support; to R. Spero, Y. Gursel, A. Abramovici, A. Cadez, M. Zucker, S. Smith, A. Bostick of Caltech, R. Butcher of Cavendish Laboratory, and R. Weiss M. Burka of MIT for their help and many useful discussions. This work was supported by the National Science Foundation under PHY. 85-04136.

When this work was done the author was on leave from Corpus Christi College of Cambridge University, U.K.

\section{References}

1. R. W. P. Drever, J. L. Hall, F. V. Kowalski, J. Hough, G. M. Ford, A. J. Munely, and H. Ward, "Laser Phase and frequency Stabilization Using an Optical Resonator," Appl. Phys. B 31, 97-105 (1983).

2. L. D. Landau and E. M. Lifshitz, Statistical Physics, 3rd Edition, Part 1, (Pergamon, New York, 1980), part. 1.

3. T. Baer, F. V. Kowalski, and J. L. Hall, "Frequency Stabilization of a 0.633- $\mu \mathrm{m} \mathrm{He}-\mathrm{Ne}$ Longitudinal Zeeman Laser," Appl. Opt. 19, 3173-3177 (1980).

4. M. A. Zumberge, "Frequency Stability of a Zeeman-Stabilized Laser," Appl. Opt. 24, 1902-1904 (1985).

5. J. Hough, D. Hils, M. D. Rayman, L-S. Ma, L. Hollberg, and J. L. Hall, "Dye-Laser Frequency Stabilization Using Optical Resonators,” Appl. Phys. B 33, 179-185 (1984).

6. J. Kauppinen, "A Simple Method for Single-Frequency Operation and Stabilization of a He-Ne Laser," Appl. Phys. B 26, 193195 (1981). 